\title{
Spacetime Noncommutativity in Models with Warped Extradimensions
}

\author{
Thorsten Ohl* $\quad$ Alexander Schenkel ${ }^{\dagger} \quad$ Christoph F. Uhlemann ${ }^{\ddagger}$ \\ Institut für Theoretische Physik und Astrophysik \\ Universität Würzburg \\ Am Hubland, 97074 Würzburg, Germany
}

July 2, 2021

\begin{abstract}
We construct consistent noncommutative (NC) deformations of the Randall-Sundrum spacetime that solve the NC Einstein equations with a non-trivial Poisson tensor depending on the fifth coordinate. In a class of these deformations where the Poisson tensor is exponentially localized on one of the branes (the NC-brane), we study the effects on bulk particles in terms of Lorentz-violating operators induced by NC-brane interactions. We sketch two models in which massive bulk particles mediate NC effects to an almostcommutative SM-brane, such that observables at high energy colliders are enhanced with respect to low energy and astrophysical observables.
\end{abstract}

\section{Introduction}

The noncommutativity (NC) of spacetime coordinates is a generic prediction of theories of quantum gravity, including string theory. If the corresponding energy scale is low enough, effects of such a NC could be observed in collider experiments 1, 2. Indeed, a consistent effective theory of a NC extension of the standard model can be constructed [3, 4, 5, 6] and detailed calculations of collider effects have been performed (see, e. g., [7, 8, 9]). However, for this to be experimentally feasible, some rather stringent constraints from low energy precision experiments and astrophysical observations (see [2] and references therein) have to be avoided.

Theories with extra spacetime dimensions provide an attractive scenario for reducing the scale of quantum gravity from the four-dimensional Planck mass and are motivated by string theory. Unfortunately, if naive dimensional analysis can be trusted, low energy and astrophysical bounds push the NC scale far above the range accessible to terrestrial colliders [2]. Therefore we need a mechanism for enhancing the collider scale NC effects relative to the low energy NC effects.

The aim of this paper is twofold: we develop a consistent description of NC in models with extra spacetime dimensions and we show how a combination of brane-localized NC, loop

\footnotetext{
*e-mail: ohl@physik.uni-wuerzburg.de

$\dagger$ e-mail: aschenkel@physik. uni-wuerzburg.de

${ }^{\ddagger}$ e-mail: uhlemann@physik. uni-wuerzburg.de
} 
factors, moderate tuning of parameters and resonance enhancement can lead to amplified NC effects at the $\mathrm{TeV}$-scale.

In section 2 we describe consistent deformations of the Randall-Sundrum background and in section 3 we study the effects of these deformations on particles propagating in the bulk. We introduce two exemplary models in section 4. While these models are not intended as complete models for physics beyond the standard model, we can use them to sketch some phenomenological options in section 5 , before concluding in section 6 .

\section{Noncommutative Randall-Sundrum background}

\section{$2.1 \quad$ Kinematical setup}

In this paper we consider NC deformations of Randall-Sundrum scenarios, which effectively reduce to a Moyal-Weyl deformation from the four-dimensional perspective. To fix our notation, we briefly introduce the classical setup known as RS1 [10]. Starting with the orbifold $\mathbb{R}^{1,3} \times S^{1} / \mathbb{Z}_{2}$ with two branes placed at the orbifold fixed-points, one obtains as a solution of the classical 5D Einstein equations a slice of $\mathrm{AdS}_{5}$ with fixed relations among the brane and bulk cosmological constants. We use the coordinates $x^{M}=\left(x^{\mu}, y\right)$ in which the RS metric takes the form

$$
d s^{2}=e^{-2 k R|y|} \eta_{\mu \nu} d x^{\mu} \otimes d x^{\nu}-R^{2} d y \otimes d y .
$$

The two branes of opposite tension are located at $y=0$ and $y=\pi$ and we refer to them as UV and IR brane, respectively.

We now construct a NC RS spacetime. Consider the following Poisson tensor on the RS background

$$
\Theta^{\mu \nu}(y)=\vartheta(|y|) \omega^{\mu \nu}, \quad \Theta^{5 \mu}=0
$$

where $\omega^{\mu \nu}$ is a general constant antisymmetric matrix and for consistency with the orbifold symmetry $\vartheta$ depends on the modulus $|y|$ only. We briefly show how to construct a $\star$-product realizing the associated $\star$-commutation relations

$$
\left[x^{\mu \star}, x^{\nu}\right]:=x^{\mu} \star x^{\nu}-x^{\nu} \star x^{\mu}=i \lambda \Theta^{\mu \nu}(y), \quad\left[x^{\mu \star}, y\right]=0 .
$$

For this, we take a (possibly degenerate) constant matrix $T_{a}{ }^{\mu}$ and the canonical Poisson tensor $\theta_{\text {can }}^{a b}$ of rank 4 in Darboux form, i.e.

$$
\theta_{\text {can }}^{a b}=\left(\begin{array}{cccc}
0 & 1 & 0 & 0 \\
-1 & 0 & 0 & 0 \\
0 & 0 & 0 & 1 \\
0 & 0 & -1 & 0
\end{array}\right)
$$

We can always find a $T_{a}{ }^{\mu}$, such that $\omega^{\mu \nu}=T_{a}{ }^{\mu} T_{b}{ }^{\nu} \theta_{\text {can }}^{a b}$ holds true. We take the set of commuting vector fields $\left\{X_{a}\right\}$ defined by

$$
X_{1}=T_{1}{ }^{\mu} \partial_{\mu}, \quad X_{2}=\vartheta(|y|) T_{2}{ }^{\mu} \partial_{\mu}, \quad X_{3}=T_{3}{ }^{\mu} \partial_{\mu}, \quad X_{4}=\vartheta(|y|) T_{4}{ }^{\mu} \partial_{\mu},
$$


and construct a $\star$-product of RJS-type [11, 12

$$
(f \star g)\left(x^{M}\right):=f\left(x^{M}\right) \exp \left(\frac{i \lambda}{2} \overleftarrow{X_{a}} \theta_{\text {can }}^{a b} \overrightarrow{X_{b}}\right) g\left(x^{M}\right),
$$

leading to the desired commutation relations (3). Note that, despite the appearance of nondifferentiable functions depending on $|y|$, (6) is a well-defined $\star$-product since the derivatives act on the coordinates $x^{\mu}$ alone.

The $\star$-product (6) is constructed in such a way that $X_{1}$ and $X_{3}$ are Killing vector fields. As shown in [13, 14, 15, these so-called semi-Killing $\star$-products (more precisely, the relevant structure is the Drinfel'd twist) have the remarkable property that the NC Einstein tensor reduces to the undeformed one. Furthermore, the classical stress-energy tensor of the RS model is a natural choice in the NC setup, since potential contributions from the $\star$-products drop out due to $4 \mathrm{D}$ translation symmetry and the semi-Killing property of the $\star$-product. Note that the brane-localized terms cause no obstructions, since the $\star$-products act on $x^{\mu}$ alone. Therefore, our NC RS background is a solution of the NC Einstein equations proposed in [16, 17] with the classical stress-energy tensor as a source. We refer to [13, 14, 15, 18, 19] for more on $\mathrm{NC}$ gravity solutions.

This completes the description of our kinematical setup. Next, we want to face the question of how to choose the up to now arbitrary function $\vartheta(|y|)$. From the NC gravity perspective we have already chosen a preferred $\star$-product of semi-Killing type, such that the classical background solves the NC Einstein equations. In this context we could further specialize to a constant $\vartheta(|y|)$ to obtain a $\star$-product constructed entirely from Killing vector fields. However, to determine $\vartheta(|y|)$ we will use a different, string theory inspired perspective, providing additional prospects for model-building.

\subsection{String theory inspired noncommutativity}

String theory with a non-vanishing background for the $B$-field arising in the massless closedstring spectrum is known to lead to $\mathrm{NC}$ effects in string scattering amplitudes (see e.g. 20 , 21, 22]). Additionally, it was shown by Seiberg and Witten [22] that a NC (super) Yang-Mills theory can be obtained in the low energy limit. The $B$-field background defines the Poisson tensor in the $\star$-product via

$$
\lambda \Theta^{\mu \nu}=2 \pi \alpha^{\prime}\left(\frac{1}{g+2 \pi \alpha^{\prime} B}\right)_{A}^{\mu \nu} .
$$

Note that we extracted the deformation parameter $\lambda$ on the left-hand-side in order to obtain a dimensionless $\Theta^{\mu \nu}$. Thus, the NC can in principle be related to the dynamics of the $B$-field using (7).

To motivate preferred choices for the $y$-dependence of the Poisson tensor we work within the supergravity approximation of string theory, dimensionally reduced to five-dimensional Anti-de-Sitter $\left(\mathrm{AdS}_{5}\right)$ space. We briefly comment on the modifications necessary for the case of a RS spacetime. The dimensional reductions are obtained via flux-compactifications of the ten-dimensional supergravities, yielding lower dimensional gauged supergravities. Of particular interest are the $N=4$ and $N=8$ gauged supergravities on $\mathrm{AdS}_{5}$ [23, 24, 25]. Both include $B$-fields in the spectrum which satisfy the same type of 'self-dual' equations of motion. Since we are only interested in the $y$-dependence of the $B$-field, we can for simplicity 
restrict ourselves to the $N=4$ theory discussed in [25]. The $B$-field equation of motion is given by

$$
\sqrt{|g|}^{-1} \epsilon^{M N R S T} \epsilon^{\alpha \beta} D_{R} B_{S T}^{\beta}=g_{1} \xi^{2} B^{\alpha M N},
$$

where $\alpha, \beta \in\{1,2\}$ label the two $B$-fields of the $N=4$ theory, $M, N, R, S$, and $T$ are 5 dimensional indices, $D_{R}$ is the covariant derivative and $\xi$ is the exponentiated dilaton. The theory admits an $\mathrm{AdS}_{5}$ solution in which $g_{1}=2 k$ is related to the curvature of the $\mathrm{AdS}_{5}$ space and $\xi=1$. In order to solve (8) on that background we make the ansatz

$$
B_{\mu \nu}^{\alpha}\left(x^{\rho}, y\right)=b_{\mu \nu}^{\alpha} \zeta^{\alpha}(y), \quad B_{5 \mu}^{\alpha}\left(x^{\rho}, y\right)=0,
$$

where $b_{\mu \nu}^{\alpha}$ is a constant antisymmetric matrix, which we parameterize by 'electric' and 'magnetic' components, i.e. we define $b_{0 i}^{\alpha}=E_{i}^{\alpha}$ and $b_{i j}^{\alpha}=\epsilon_{i j}{ }^{k} B_{k}^{\alpha}$. Plugging this into (8) we obtain

$$
\zeta^{1}(y)=a_{1} e^{k R y}+a_{2} e^{-k R y}, \quad \zeta^{2}(y)=a_{1} e^{k R y}-a_{2} e^{-k R y},
$$

and $E_{i}^{2}=B_{i}^{1}, B_{i}^{2}=-E_{i}^{1}$, i.e. $b_{\mu \nu}^{2}$ is fixed in case $b_{\mu \nu}^{1}$ is given, or vice versa. The relevant object entering the Poisson tensor is a linear combination of the two $B$-fields 1 , which we denote by

$$
2 \pi \alpha^{\prime} B_{\mu \nu}(y)=a_{+} e^{k R y} b_{\mu \nu}^{+}+a_{-} e^{-k R y} b_{\mu \nu}^{-} .
$$

Note that we have defined for later convenience the parameters $a_{ \pm}$and $b_{\mu \nu}^{ \pm}$to be dimensionless by extracting the factor $2 \pi \alpha^{\prime}$ and that the $b_{\mu \nu}^{ \pm}$are not independent. For the case of a RS spacetime (11) is the solution on the fundamental domain. The lifting to the covering space is obtained by periodical continuation with appropriate parity and fine-tuned brane-localized $B$-field mass terms.

To understand the $y$-dependence of the Poisson tensor (7), we consider the special cases $B_{\mu \nu}^{ \pm}(y):=\left.B_{\mu \nu}(y)\right|_{a_{\mp}=0}$ separately. Assuming $\vec{E}_{ \pm} \cdot \vec{B}_{ \pm}=0$, which is a necessary requirement for an effective field theory description [27, we derive from (7) the Poisson tensor

$$
\lambda \Theta_{ \pm}^{\mu \nu}=-2 \pi \alpha^{\prime} \frac{a_{ \pm} e^{(4 \pm 1) k R y}}{a_{ \pm}^{2} e^{2(2 \pm 1) k R y}\left(\vec{B}_{ \pm}^{2}-\vec{E}_{ \pm}^{2}\right)+1} b_{ \pm}^{\mu \nu} \approx-\frac{2 \pi \alpha^{\prime}}{a_{ \pm}\left(\vec{B}_{ \pm}^{2}-\vec{E}_{ \pm}^{2}\right)} b_{ \pm}^{\mu \nu} e^{\mp k R y}
$$

where $b_{ \pm}^{\mu \nu}:=\eta^{\mu \rho} \eta^{\nu \sigma} b_{\rho \sigma}^{ \pm}$is defined via the flat metric $\eta^{\mu \nu}$ and the last approximation holds for not unnaturally small $a_{ \pm}$and $\vec{B}_{ \pm}^{2}-\vec{E}_{ \pm}^{2}$. Even though it was shown in [27] that a unitary NC QFT is obtained for $\vec{B}^{2} \geq \vec{E}^{2}$, we restrict our discussion to the case $\vec{B}^{2}>\vec{E}^{2}$, known as space-like NC.

In the following we assume that the Poisson tensor calculated above will enter as a $\star$ product (6) in the effective field theory description of the physics in a $B$-field background. Note that this is only a physical assumption which we do not derive here from a more fundamental theory. The emergence of $\star$-products in effective field theories due to a constant $B$-field was shown by Seiberg and Witten for the case of Yang-Mills theories [22]. For the low energy gravity sector of string theory in a $B$-field background see [28].

\footnotetext{
${ }^{1}$ See [26], where the bosonic sector of the $N=4$ theory was obtained by means of a reduction ansatz from the ten-dimensional type IIB supergravity. The ansatz used there shows that the combination $\hat{B}+i \hat{C}_{2}$, of the ten-dimensional NS-NS sector B-field $\hat{B}$ and R-R sector gauge field $\hat{C}_{2}$, is determined by $B^{1}+i B^{2}$.
} 


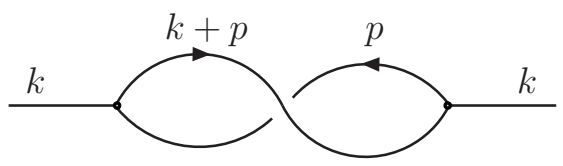

Figure 1: Sample diagram for the bulk particle self-energies due to NC brane matter. Diagrams of this type induce Lorentz-violating effective two-point operators for the bulk particle.

Next, we have to set the scales of our effective field theory description. Since we have a vanishing dilaton background, we identify $\alpha^{\prime}$ with the five-dimensional Planck length, $\alpha^{\prime}=\hat{l}_{P}^{2}$, which will be $\mathcal{O}\left(\mathrm{TeV}^{-2}\right)$. Due to the exponential $y$-dependence of $\lambda \Theta_{ \pm}^{\mu \nu} \sqrt{12}$, the resulting $y$-dependent $\mathrm{NC}$ scale can be taken to be $\mathrm{TeV}$-scale on one brane, while being exponentially small on the other brant ${ }^{2}$. Because of the exponentially strong localization of the Poisson tensor towards one of the branes, we can, as a simplifying approximation, restrict $\Theta^{\mu \nu}$ to the brane where it is localized and assume the remaining part of the theory to be commutative. We call this the NC-brane approximation.

\section{$3 \quad \mathrm{NC}$ effects on bulk particles}

Before considering explicit models utilizing the setup described above, we study the NC effects on bulk particles due to the coupling to matter on the NC brane in a general context. We use the NC-brane approximation throughout this section. Ultimately, the SM will be confined to the almost commutative brane and $\mathrm{NC}$ effects are communicated from the NC brane to the SM brane via the bulk particles (e.g. the KK graviton). We are thus interested in the effect of the NC sector on bulk particles, which is the radiative induction of Lorentz-violating effective operators. We study these operators for the radion and the graviton as well as for a bulk $\mathrm{U}(1)$ gauge field at the one-loop level. For this we first classify all possible lowest-dimensional Lorentz-violating effective operators arising from NC interactions and then show that they are indeed induced via nonplanar self-energies of the type shown in Fig. 1 and simple matter models on the NC brane. In planar diagrams, the phases arising due to the $\star$-product cancel, while the nonplanar amplitudes are proportional to $\cos (\lambda k \Theta p)$. We expand the integrand in $\lambda$ up to the leading order providing NC effects, which is $\mathcal{O}\left(\lambda^{2}\right)$. Kaluza-Klein reduction is not obstructed by the $\star$-product due to $\left[x^{\mu}, y\right]=0$, and is applied throughout.

Radion and graviton Applying a graviton expansion to the five-dimensional Einstein Hilbert action on the $\mathrm{AdS}_{5}$ background with two branes and using suitable gauge fixings to extract and decouple the physical degrees of freedom, the Kaluza-Klein expanded gravity action reads [29, 30]

$$
\begin{aligned}
S_{\text {bulk }} & =\frac{1}{2} \int d^{4} z \partial_{\mu} \phi \partial^{\mu} \phi+\frac{1}{4} \sum_{n} \int d^{4} z\left(\partial_{\mu} u_{\nu \rho}^{(n)} \partial^{\mu} u^{(n)^{\nu \rho}}-m_{n}^{2} e^{2 k R \pi} u_{\nu \rho}^{(n)} u^{(n)^{\nu \rho}}\right), \\
S_{\text {NC-brane }}^{\text {int }} & =\sum_{n} \frac{\kappa_{n}}{2} \int d^{4} z u_{\mu \nu}^{(n)} T_{\star}^{\mu \nu}+\frac{\kappa_{\phi}}{2 \sqrt{3}} \int d^{4} z \phi T_{\star \mu}^{\mu},
\end{aligned}
$$

\footnotetext{
${ }^{2}$ Since the Poisson tensor is of dimension (length) $)^{-2}$, the effective NC scale on the other brane is suppressed roughly by a factor $e^{\frac{1}{2} k R \pi}$.
} 
where $\kappa_{n}$ and $\kappa_{\phi}$ are coupling constants of dimension -1 , which are related to the fivedimensional Planck mass, overlaps of the KK gravitons and the radion with the NC brane and exponential factors $e^{k R \pi}$. These relations will be discussed in detail later. $z^{\mu}$ denotes coordinates which are Galilean on the SM brane, i.e. $z^{\mu}=e^{-k R \pi} x^{\mu}, \phi$ denotes the radion and $u_{\mu \nu}^{(n)}$ are the graviton KK modes. By cyclicity of the integral, in each term one of the $\star-$ products can be replaced by an ordinary product. Thus the $\star$-products drop out of the quadratic part of the action and all $\mathrm{NC}$ effects can be absorbed into the definition of the deformed stress-energy tensor $T_{\star}^{\mu \nu}$ on the NC brane.

We now consider the lowest-dimensional Lorentz-violating effective operators for the radion field. Assuming for simplicity massless matter fields on the NC brane, we obtain

$$
S_{\mathrm{eff}}^{\mathrm{s}}=\frac{\left(\kappa_{\phi} \lambda\right)^{2}}{2} \int d^{4} z a_{1}^{\mathrm{s}} \phi \tilde{\partial}^{2} \partial^{6} \phi,
$$

where $\left(\kappa_{\phi} \lambda\right)^{2}$ has dimension $-6, a_{1}^{\mathrm{s}}$ is dimensionless and $\tilde{\partial}^{\mu}:=\Theta^{\mu \nu} \partial_{\nu}$. Note that, if we had considered massive fields on the $\mathrm{NC}$ brane, the operator as given above with any of the $\partial^{2}$ replaced by the squared mass would also be possible. To show by an explicit example that this operator is indeed induced, we consider an abelian gauge field $A_{\mu}$ and a massless real scalar $\chi$ on the NC brane, described by

$$
\begin{aligned}
S_{\text {NC-brane }} & =-\frac{1}{4} \int d^{4} z F^{\mu \nu} F_{\mu \nu}+\frac{1}{2} \int d^{4} z \partial_{\mu} \chi \partial^{\mu} \chi, \\
T_{\star}^{\mu \nu} & =F^{\mu \rho} \star F^{\nu}{ }_{\rho}-\frac{1}{4} \eta^{\mu \nu} F^{\rho \sigma} \star F_{\rho \sigma}-\partial^{\mu} \chi \star \partial^{\nu} \chi+\frac{\eta^{\mu \nu}}{2} \partial_{\rho} \chi \star \partial^{\rho} \chi .
\end{aligned}
$$

We calculate the nonplanar radion self-energy $\Pi(k)$ due to the NC-brane scalar field (see Fig. 11 using dimensional regularization and find $\Pi(k)=a_{1}^{\mathrm{s}} \frac{i}{\epsilon} \kappa_{\phi}^{2} \lambda^{2} k^{6} \tilde{k}^{2}+$ finite, with $a_{1}^{\mathrm{s}} \neq 0$. This shows that the expected operator is indeed induced for a simple matter model on the NC brane. The self-energy due to the NC-brane vector field vanishes, since the corresponding part of the stress-energy tensor is traceless.

Next, we consider the graviton. The four-dimensional gauge transformations of the massless graviton mode $u_{\mu \nu}^{(0)}$ are given by

$$
u_{\mu \nu}^{(0)} \rightarrow u_{\mu \nu}^{(0)^{\prime}}=u_{\mu \nu}^{(0)}-\partial_{\mu} \zeta_{\nu}-\partial_{\nu} \zeta_{\mu} .
$$

Noting that both $\tilde{\partial}^{\mu} \tilde{\partial}^{\nu} u_{\mu \nu}^{(0)}$ and the linearized scalar curvature $R=\kappa_{0}\left(\eta^{\mu \nu} \partial^{\rho} \partial_{\rho}-\partial^{\mu} \partial^{\nu}\right) u_{\mu \nu}^{(0)}+$ $\mathcal{O}\left(\kappa_{0}^{2}\right)=: \kappa_{0} D^{\mu \nu} u_{\mu \nu}^{(0)}+\mathcal{O}\left(\kappa_{0}^{2}\right)$ are gauge invariant, we can write the following gauge invariant and Lorentz-violating effective operators for the graviton

$$
\begin{aligned}
\mathcal{S}_{\mathrm{eff}}^{\mathrm{grav}}=\frac{\left(\kappa_{n} \lambda\right)^{2}}{4} \int d^{4} z u_{\mu \nu}^{(n)}( & c_{1} D^{\mu \nu} \tilde{\partial}^{\rho} \tilde{\partial}^{\sigma} \partial^{2} \\
& \left.+c_{2} D^{\mu \rho} \tilde{\partial}^{\nu} \tilde{\partial}^{\sigma} \partial^{2}+c_{3} D^{\mu \nu} D^{\rho \sigma} \tilde{\partial}^{2}+c_{4} D^{\mu \rho} D^{\nu \sigma} \tilde{\partial}^{2}\right) \partial^{2} u_{\rho \sigma}^{(n)}
\end{aligned}
$$

These are all possible effective operators, because in one-loop diagrams the Poisson tensor is always contracted with one external momentum. Furthermore, four-dimensional gauge invariance restricts the possible loop-induced operators for all graviton modes, not just for the massless mode. Indeed, the structure of the couplings is the same for massless and 
massive modes. Therefore, if an effective operator for a higher KK graviton is induced by a self-energy diagram as in Fig. 1, the same divergence appears in the massless graviton selfenergy. Consequently, the effective graviton operators are restricted to those of (17) for all KK modes.

Explicitly calculating the nonplanar graviton self-energies $\Pi_{\mathrm{s}}^{\mu \nu \rho \sigma}(k)$ and $\Pi_{\mathrm{v}}^{\mu \nu \rho \sigma}(k)$ in the simple matter model of a NC-brane scalar and vector field, respectively, we find ${ }^{3}$

$$
\Pi_{\mathrm{s} / \mathrm{v}}^{\mu \nu \rho \sigma}(k)=\frac{i}{\epsilon} \kappa_{n}^{2} \lambda^{2} \sum_{i=1}^{4} c_{i}^{\mathrm{s} / \mathrm{v}} O_{i}^{\mu \nu \rho \sigma}(k)+\text { finite },
$$

where all the $c_{i}^{\mathrm{s}}$ and $c_{i}^{\mathrm{v}}$ are nonzero and $O_{i}^{\mu \nu \rho \sigma}(k)$ are the momentum-space versions of the operators in (17), as given in App. A. Hence, all gauge invariant Lorentz-violating effective operators given above are induced radiatively. We find that the $\Pi_{\mathrm{S}, \mathrm{v}}^{\mu \nu \rho \sigma}(k)$ fulfill the standard Ward identity $k_{\mu} \Pi_{\mathrm{s}, \mathrm{v}}^{\mu \nu \rho \sigma}(k)=0$ (and the ones arising from permutations of the indices of $\left.\Pi_{\mathrm{s}, \mathrm{v}}^{\mu \nu \rho \sigma}(k)\right)$, which can be understood from the fact that the action 13 consists of free gravitons coupled to a conserved $\star$-deformed matter current $T_{\star}^{\mu \nu}$. Whether these Ward identities continue to hold at higher orders in the gravitational coupling, or whether they have to be deformed as well, is still an open question, because it is in general not possible to absorb all $\mathrm{NC}$ effects into a deformed matter stress-energy tensor $T_{\star}^{\mu \nu}$ in this case.

Abelian gauge field We now turn to a bulk U(1) gauge field $A_{M}(M=0,1,2,3,5)$, which we assume to be coupled to a charged scalar $\chi$ on the NC brane (the fields $A_{\mu}$ and $\chi$ are unrelated to those of the previous paragraph). After gauge fixing and Kaluza-Klein expansion $A_{\mu / 5}(z, y)=\sum_{n} A_{\mu / 5}^{(n)}(z) t_{1 / 2}^{(n)}(y)$ the action is given by

$$
\begin{aligned}
S_{\text {bulk }}= & \frac{1}{2} \sum_{n} \int d^{4} z A_{\mu}^{(n)}\left(\left(\partial^{2}+m_{n}^{2}\right) \eta^{\mu \nu}-\left(1-\frac{1}{\xi}\right) \partial^{\mu} \partial^{\nu}\right) A_{\nu}^{(n)} \\
& -\frac{1}{2} \sum_{n} \int d^{4} z A_{5}^{(n)}\left(\partial^{2}+\xi m_{n}^{2}\right) A_{5}^{(n)}, \\
S_{\text {NC-brane }}= & \int d^{4} z\left(D_{\mu} \chi\right)^{\dagger} \star D^{\mu} \chi, \quad D_{\mu} \chi=\partial_{\mu} \chi+i \sum_{n} g_{n} A_{\mu}^{(n)} \star \chi .
\end{aligned}
$$

We choose Neumann/Dirichlet boundary conditions for $A_{\mu} / A_{5}$, and apply unitary gauge to decouple the massive scalar modes. The dimensionless coupling constants $g_{n}$ contain the gauge coupling $g_{5}$ and the brane-overlap $t_{1}^{(n)}\left(y_{\text {NC-brane }}\right)$. The four-dimensional gauge transformations are given by $A_{\mu}^{(0)} \rightarrow A_{\mu}^{(0)}+\partial_{\mu} \omega$ and, as explained above, restrict the possible loop-induced effective operators for all KK modes. Using the restrictions from gauge invariance, we find the following two independent Lorentz-violating effective operators

$$
S_{\text {eff }}^{\mathrm{A}}=\frac{\left(g_{n} \lambda\right)^{2}}{4} \int d^{4} z F_{\mu \nu}^{(n)}\left(d_{1} \Theta^{\mu \rho} \Theta^{\nu \sigma} \partial^{4}+d_{2} \eta^{\mu \rho} \eta^{\nu \sigma} \tilde{\partial}^{2} \partial^{2}\right) F_{\rho \sigma}^{(n)},
$$

where $F_{\mu \nu}^{(n)}=\partial_{[\mu} A_{\nu]}^{(n)}$.

\footnotetext{
${ }^{3}$ The brane-localized interactions introduce mixing between the KK modes which, however, will be ignored in the following since it is not relevant to our investigations.
} 


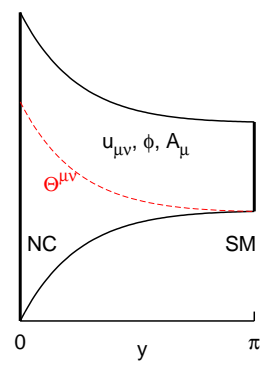

(a)

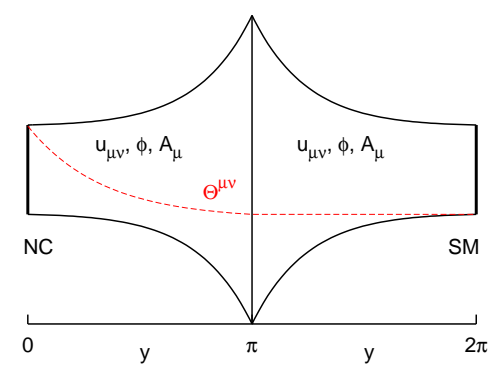

(b)

Figure 2: The basic setups for our two models. (a) shows the standard RS setup with NC UV brane, (b) shows the three-brane model with the second NC IR brane.

Using the simple NC brane matter model (19b), we find for the nonplanar gauge boson self-energy $\Pi^{\mu \nu}(k)=\frac{i}{\epsilon} g_{n}^{2} \lambda^{2}\left(d_{1} O_{1}^{\mu \nu}(k)+d_{2} O_{2}^{\mu \nu}(k)\right)+$ finite, where $O_{i}^{\mu \nu}(k)$ are the momentumspace versions of the operators given in $(20)$ and $d_{1}, d_{2} \neq 0$. This shows that both Lorentzviolating effective operators are induced at the one-loop level.

\section{Explicit models}

We now discuss two explicit models which realize the idea of transmitting NC effects via bulk particles from a NC sector to the SM. In both models the SM is confined to the IR brane where the $B$-field solution is chosen such that spacetime NC is exponentially suppressed. As explained in Section 2.2, we apply the NC-brane approximation after constructing the models and assume the bulk and the SM brane to be commutative. The general effects of a NC brane on bulk particles have been discussed in the previous section, so the remaining task is to determine the coupling strengths of the bulk particles to the different branes. While the localizations of the KK gravitons and the radion are fixed by the geometry, leading to fixed coupling constants $\kappa_{\phi}$ and $\kappa_{n}$ in $(13)$, the bulk gauge field offers additional freedom to fix its localization, e.g. via boundary kinetic/mass terms.

The setup for the two models we are considering is depicted in Fig. 2, In the first part we discuss the standard RS setup with a UV-brane localized NC (see Fig. 2(a)) and in the second part we use an extended RS scenario, where the NC is localized on a second IR brane (see Fig. 2(b)).

\subsection{NC Randall-Sundrum model}

We consider the standard RS setup shown in Fig. 2(a). The $B$-field configuration is chosen as $B_{\mu \nu}^{+}$, implying the Poisson tensor $\Theta_{+}^{\mu \nu}$ of $(12)$, such that the IR brane, where the SM is localized, is almost commutative, while the UV brane is not. To determine the brane couplings of the KK gravitons and radion we require their mode functions entering the KK reduction 
of the five-dimensional action. The KK ansatz and the mode functions are given by [29, 30]

$$
\begin{aligned}
& u_{\mu \nu}(x, y)=\sum_{n} u_{\mu \nu}^{(n)}(x) f_{n}(y), \quad \int d y e^{2 k R|y|} f_{m}(y) f_{n}(y)=\delta_{n m}, \\
& f_{0}(y)=N_{0} e^{-2 k R|y|}, \quad f_{n}(y)=N_{n}\left(Y_{1}\left(\gamma_{n}\right) J_{2}\left(\gamma_{n} e^{k R|y|}\right)-J_{1}\left(\gamma_{n}\right) Y_{2}\left(\gamma_{n} e^{k R|y|}\right)\right),
\end{aligned}
$$

where $J_{1}\left(\gamma_{n} e^{k R \pi}\right)=0$ and $m_{n}=\gamma_{n} k$.

The coupling of gravity to the SM brane at $y=\pi$ is given by 13 with $\kappa_{n / \phi}=\kappa_{n / \phi}^{\mathrm{SM}}$. We define $\kappa_{\mathrm{SM}}:=\kappa_{0}^{\mathrm{SM}}$ as the coupling of the massless graviton mode to SM matter, which has to be fixed to $\kappa_{\mathrm{SM}}=\sqrt{16 \pi} M_{\text {Planck }}^{-1}$ to reproduce the observed four-dimensional gravitational coupling. One obtains the relations

$$
\kappa_{0}^{\mathrm{SM}}=\hat{\kappa} f_{0}(\pi) e^{k R \pi}, \quad \kappa_{n}^{\mathrm{SM}}=\kappa_{\mathrm{SM}} \frac{f_{n}(\pi)}{f_{0}(\pi)}, \quad \kappa_{\phi}^{\mathrm{SM}}=\kappa_{\mathrm{SM}} e^{k R \pi} .
$$

The five-dimensional gravitational coupling $\hat{\kappa}$ is chosen such that $\kappa_{\mathrm{SM}}=\sqrt{16 \pi} M_{\text {Planck }}^{-1}$, i.e. $\hat{\kappa}=$ $\kappa_{\mathrm{SM}} e^{k R \pi} / N_{0}$. For a typical value of $k R \approx 11$ the first $\mathrm{KK}$ mode of the graviton has a mass of order $\mathrm{TeV}$ and a SM-brane coupling of order $\mathrm{TeV}^{-1}$. The radion couples to the SM brane with a similar strength as the massive gravitons. where

The coupling to the UV brane, localized at $y=0$, is given by 13 with $\kappa_{n / \phi}=\kappa_{n / \phi}^{\mathrm{UV}}$,

$$
\kappa_{n}^{\mathrm{UV}}=\hat{\kappa} f_{n}(0) e^{-k R \pi}, \quad \kappa_{\phi}^{\mathrm{UV}}=\kappa_{\mathrm{SM}} e^{-k R \pi} .
$$

Note that this is different from the couplings derived in [29, 30] for a UV-brane observer, since we describe the UV-brane physics as seen from the IR brane, inducing the additional redshift factor $e^{-k R \pi}$.

The massive gravitons are of phenomenological interest due to their enhanced couplings to the SM brane. However, from expressions (22) and (23) we find that the coupling of the massive gravitons to the NC brane is exponentially suppressed. Thus, the Lorentz-violating operators (17) are Planck-scale suppressed and their effect for SM physics at the TeV scale is negligible. The same applies to the radion.

In contrast to the graviton and radion where the boundary conditions are fixed by the presence of the branes, the localization of a bulk vector field can be adjusted by a suitable choice of boundary conditions and brane-localized mass and/or kinetic terms. We will therefore employ an abelian bulk gauge field here and realize observable NC effects via the graviton in the next section in an extended RS setup. To the bulk and NC-brane actions of (19) we add the IR-brane action

$$
S_{\text {IR-brane }}=\int d^{4} z\left(D^{\mu} \phi D_{\mu} \phi+V(\phi)+\mathcal{L}_{\mathrm{SM}}\right), \quad D_{\mu} \phi=\partial_{\mu} \phi+i \sum_{n} g_{5} A_{\mu}^{(n)} t_{1}^{(n)}(\pi) \phi,
$$

where $\mathcal{L}_{\mathrm{SM}}$ denotes the Lagrangian of the SM and possible interactions with the bulk gauge field. The scalar $\phi$ is charged under the bulk $\mathrm{U}(1)$ and $V(\phi)$ is such that $\langle\phi\rangle=v$ spontaneously breaks the bulk gauge symmetry. We then find the brane-localized mass term $-g_{5}^{2} v^{2} A_{\mu}^{(n)} A_{\nu}^{(m)} \eta^{\mu \nu} t_{1}^{(n)}(\pi) t_{1}^{(m)}(\pi) \delta(y-\pi)$. With Neumann boundary condition on the NC brane the KK wave functions $t_{1}^{(n)}$ of $A_{\mu}^{(n)}$ are given by

$$
t_{1}^{(n)}(y)=N_{n} e^{k R(y-\pi)}\left(J_{1}\left(\frac{m}{k} e^{k R(y-\pi)}\right) Y_{0}\left(\frac{m}{k}\right)-Y_{1}\left(\frac{m}{k} e^{k R(y-\pi)}\right) J_{0}\left(\frac{m}{k}\right)\right) .
$$


Focussing on the lightest KK mode, we find from the IR-brane boundary condition $t_{1}^{(n)^{\prime}}$ $\left.2 R g_{5}^{2} v^{2} t_{1}^{(n)}\right|_{y=\pi}=0$ a mass of order TeV. Furthermore, the lightest mode is almost delocalized, with appreciable coupling to both branes. It can therefore transmit NC effects to the SM brane.

\subsection{Extended NC Randall-Sundrum model}

The second model realizes NC effects via the KK gravitons. The basic setup is depicted in Fig. 2(b), As in the standard RS setup we consider the orbifold $\mathbb{R}^{1,3} \times S^{1} / \mathbb{Z}_{2}$. We parameterize the circle by $y$ running from $-2 \pi$ to $2 \pi$. Two branes of tension $\Lambda_{1}, \Lambda_{3}$ are placed at the orbifold fixed-points $y_{1}=0, y_{3}=2 \pi$ and a third one of tension $\Lambda_{2}$ at $y_{2}=\pi$. We solve the associated Einstein equations

$$
R_{M N}-\frac{1}{2} g_{M N} R=-\frac{1}{4 M_{5}^{3}}\left(\Lambda g_{M N}+\sum_{i=1}^{3} \Lambda_{i} \delta\left(y-y_{i}\right) \frac{\sqrt{-\hat{g}^{(i)}}}{\sqrt{g}} \hat{g}_{\mu \nu}^{(i)} \delta_{M}^{\mu} \delta_{N}^{\nu}\right),
$$

where $\hat{g}^{(i)}$ denotes the induced metric on the $i$-th brane. With the ansatz $d s^{2}=e^{-2 \sigma(\hat{y})} \eta_{\mu \nu} d x^{\mu} \otimes$ $d x^{\nu}-R^{2} d y \otimes d y$, where $\hat{y}(y)=|| y|-\pi|$ is consistent with the orbifold symmetry, we find the solution $\sigma(\hat{y})=k R \hat{y}$, where $k$ is defined by $\Lambda=-24 M_{5}^{3} k^{2}$, and $\Lambda_{1}=\Lambda_{3}=\Lambda / k, \Lambda_{2}=-\Lambda_{1}$. We thus find two standard RS backgrounds glued together at the UV brane.

The $B$-field configuration is chosen as $B_{\mu \nu}^{-}(\pi-y)$ for $y \in[0, \pi]$ and $B_{\mu \nu}^{+}(y-\pi)$ for $y \in$ $(\pi, 2 \pi]$, such that $B$ is continuous at the UV brane at $y=\pi$. By this choice, we obtain the continuous Poisson tensor $\Theta_{-}^{\mu \nu}(\pi-y)$ for $y \in[0, \pi]$ and $\Theta_{+}^{\mu \nu}(y-\pi)$ for $y \in(\pi, 2 \pi]$, see $(12)$, which exponentially grows towards the IR brane at $y=0$. This yields in the NC-brane approximation a commutative IR brane at $y=2 \pi$ where the SM is located, a commutative UV brane at $y=\pi$, and a NC IR brane at $y=0$.

Performing the graviton expansion $u_{\mu \nu}(x, y)=\sum_{n} u_{\mu \nu}^{(n)}(x) \hat{f}_{n}(y)$, we find $\hat{f}_{n}(y)=f_{n}(\hat{y})$ with $f_{n}$ given in (21b). The masses of the graviton modes and their matter couplings on the SM and UV brane are equal to those of the standard RS case. The matter couplings on the $\mathrm{NC}$ brane are equal to those on the $\mathrm{SM}$ brane, i.e. of order $\mathrm{TeV}^{-1}$ for the massive modes. Thus, the phenomenology of this setup is very similar to that of the standard RS scenario, but we do have an effective communication of Lorentz-violating effects from the NC brane to the SM brane via gravity.

\section{Options for phenomenology}

In the previous section we have constructed two models with an effective transmission of Lorentz violation from the NC brane to the SM brane. The main difference is the messenger particle, which is a massive vector field in the first one and the massive graviton in the second. As an illustration, we discuss in this section an exemplary collider process and a way to compare our setup to experimental constraints on the NC scale.

\subsection{NC effects in scattering processes}

We consider the scattering of two massless quarks $q$, producing two SM photons $\gamma$ in the extended RS model. In addition to the s-channel exchange of the massive graviton, $\bar{q} q \rightarrow$ $u_{\mu \nu}^{(n)} \rightarrow \gamma \gamma$, we have the $\mathcal{O}\left(\lambda^{2}\right)$ Lorentz-violating contributions shown in Fig. $3(\mathrm{a})$. 


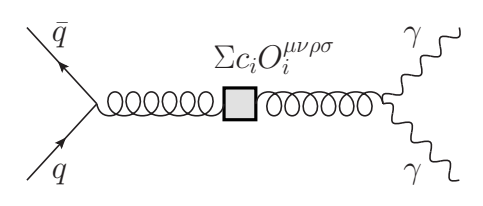

(a)

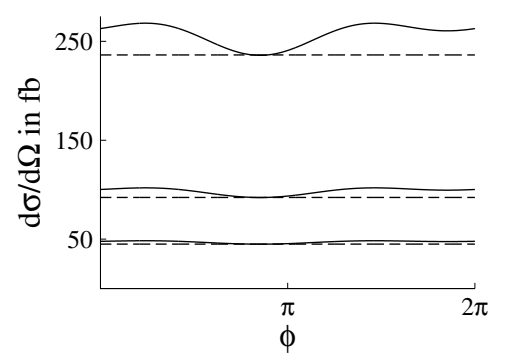

(b)

Figure 3: (a) Lorentz-violating contribution to $\bar{q} q \rightarrow \gamma \gamma$ in the three-brane model. (b) Cross section for $\bar{q} q \rightarrow \gamma \gamma$ scattering with a polar angle of $\pi / 3$ and $\sqrt{s} \in\{0.985,0.990,0.995\} \mathrm{TeV}$. Dashed lines show the cross section for the commutative case $\lambda=0$, solid lines for $\lambda=$ $0.1 \mathrm{TeV}^{-2}$, see $(27)$. The SM contributions are ignored, since they are subdominant at the resonance.

The relevant model parameters are the mass and width of the first KK graviton mode and its coupling to the SM and NC brane. We fix them by the reasonable choice [31]

$$
m_{1}=1 \mathrm{TeV}, \quad \kappa_{1}^{S M}=0.1 \beta_{1} \mathrm{TeV}^{-1}, \quad \Gamma_{1}=0.01 \mathrm{TeV}, \quad \lambda=0.1 \mathrm{TeV}^{-2},
$$

where $\beta_{1}=3.83$ is the first root of $J_{1}$.

Due to the Lorentz-violating operators, the cross section shows the typical azimuthalangle dependence, as shown in Fig. $3(\mathrm{~b})$. This dependence is due to the $\vec{E}$ components of the Poisson tensor. We find a similar effect in the scattering of vector bosons, and also in the standard RS model for cross sections involving the Lorentz-violating contribution from the massive bulk vector field. The main feature of our models is the amplification of the $\mathrm{NC}$ effects at the resonance, while they are strongly suppressed outside the resonance region. This offers the possibility to detect - together with the additional massive particles due to the extradimensional setup - effects of spacetime $\mathrm{NC}$ in particle collisions at the $\mathrm{TeV}$ scale.

\subsection{NC effects on the SM photon}

Comparing our models to experimental bounds from laboratory experiments and astrophysics is possible by considering the effective Lorentz-violating operators which are induced for the SM photon propagator. In our models, such operators are induced by the photon self-energy due to the massive bulk particle with effective-operator insertions. Hence, they effectively are an (at least) two-loop effect and there is no resonance enhancement, implying strong suppression compared to the effect on scattering cross sections.

We first discuss the second model, where the graviton mediates NC and the photon is affected by the presence of NC interactions by the self-energy diagrams shown in Fig. 4. The Lorentz-violating operators at lowest order $\mathcal{O}\left(\lambda^{2}\right)$ are

$$
S_{\text {eff }}^{\gamma}=\kappa_{n}^{4} \lambda^{2} \int d^{4} z F_{\mu \nu}\left(d_{1}^{\gamma} \Theta^{\mu \rho} \Theta^{\nu \sigma} m_{n}^{8}+d_{2}^{\gamma} \eta^{\mu \rho} \eta^{\nu \sigma} m_{n}^{6} \tilde{\partial}^{2}+d_{3}^{\gamma} \Theta^{\mu \lambda} \Theta_{\lambda}{ }^{\rho} \eta^{\nu \sigma} m_{n}^{8}\right) F_{\rho \sigma},
$$

and those with $m_{n}^{2}$ replaced by $\partial^{2}$. However, the relevant operators for laboratory experiments at low energy and for astrophysics, where photons are on-shell, are those with a minimal 

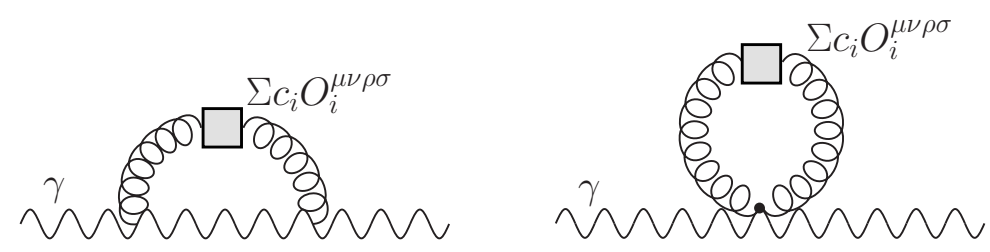

Figure 4: NC contributions to the photon self-energy in the extended RS model.

number of derivatives. We calculate the Lorentz-violating divergent part of the diagrams in Fig. 4 , i.e. the $\beta$-functions of the $d_{i}^{\gamma}$, and find it proportional to the third operator in $(28)$ and $3 c_{1}+17 c_{2}-45 c_{4}$. The coefficient $c_{3}$ does not appear since, with $B_{\mu \nu \rho \sigma}(p)$ denoting the numerator of the massive graviton propagator, $B_{\mu \nu \mu^{\prime} \nu^{\prime}}(p) O_{3}^{\mu^{\prime} \nu^{\prime} \rho^{\prime} \sigma^{\prime}}(p) B_{\rho^{\prime} \sigma^{\prime} \rho \sigma}(p) \propto\left(p^{2}-m^{2}\right)^{2}$ cancels the two massive propagator denominators, leaving only scale-free integrals. This linear combination of the $c_{i}$ can be chosen to vanish, such that $d_{i}^{\gamma}=0$ can be fixed at laboratory energy scales and is preserved by renormalization group running at leading order. We note that this does not affect the azimuthal-angle dependence in the scattering cross section, which depends on $c_{2}$ only. A detailed discussion of experimental bounds on the entries of $\kappa^{\mu \nu \rho \sigma}$ in $-\frac{1}{4} \kappa^{\mu \nu \rho \sigma} F_{\mu \nu} F_{\rho \sigma}$ can be found in [32, 33].

In the first model, the situation is even better. Since the SM photon is not charged under the bulk U(1) gauge group, it is affected starting effectively at the three-loop order, and we expect an additional suppression in that case.

\section{Conclusion}

We have studied deformations of the Randall-Sundrum spacetime induced by a Poisson tensor depending on the radial coordinate $y$, and constructed a semi-Killing $\star$-product realizing the associated commutation relations. Due to the semi-Killing property, the classical RandallSundrum metric solves the NC Einstein equations for generic $y$-dependence. Exploiting the relation of the Poisson tensor to the B-field as motivated by string theory in the low-energy description in terms of gauged supergravity on $\mathrm{AdS}_{5}$, we found preferred choices for the radialcoordinate dependence where the Poisson tensor is exponentially localized towards either the UV or IR brane.

Assuming the Poisson tensor to be confined to one of the branes, we classified the effective Lorentz-violating operators arising from brane-localized NC interactions for bulk particles, namely the graviton, radion and a bulk $\mathrm{U}(1)$ gauge field. Choosing simple matter models on the NC brane, we have shown that these operators are induced radiatively. We then constructed two models with an effective transmission of $\mathrm{NC}$ effects to an (almost) commutative SM brane via bulk particles. In the standard RS setup we had to employ a bulk gauge field, while in an extended model with three branes the massive gravitons are well suited.

As a phenomenological illustration we gave a scattering cross section which - close to the bulk-particle resonance - shows the typical azimuthal-angle dependence, and briefly studied the effective operators induced for the SM photon. This calls for more detailed model building and phenomenological studies, which, however, is beyond the scope of this work. 


\section{Acknowledgements}

We thank Paolo Aschieri and Thomas Flacke for valuable discussions and comments. AS is supported by Deutsche Forschungsgemeinschaft through the Research Training Group GRK 1147 Theoretical Astrophysics and Particle Physics. CFU is supported by the German National Academic Foundation (Studienstiftung des deutschen Volkes) and by Deutsche Forschungsgemeinschaft through the Research Training Group GRK 1147 Theoretical Astrophysics and Particle Physics.

\section{A Feynman rules}

For explicitness we list the additional $\mathcal{O}\left(\lambda^{2}\right)$ Feynman rules for the bulk-particle KK modes and give the momentum-space expressions of the effective two-point operators $O_{i}^{\mu \nu \rho \sigma}(k)$ for the graviton and $O_{i}^{\mu \nu}(k)$ for the bulk gauge field:

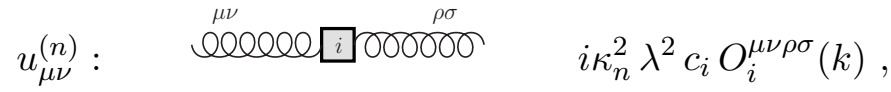

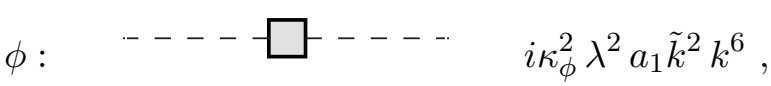

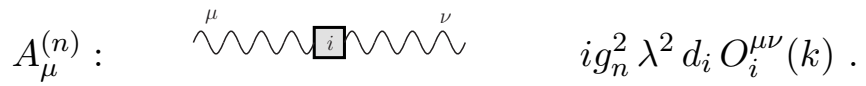

The effective operators are $O_{1}^{\mu \nu}(k)=k^{4} \tilde{k}^{\mu} \tilde{k}^{\nu}, O_{2}^{\mu \nu}(k)=\tilde{k}^{2} k^{2}\left(k^{\mu} k^{\nu}-\eta^{\mu \nu} k^{2}\right)$ and

$$
\begin{aligned}
& O_{1}^{\mu \nu \rho \sigma}(k)=\operatorname{Sym}\left[k^{4}\left(k^{\mu} k^{\nu}-\eta^{\mu \nu} k^{2}\right) \tilde{k}^{\rho} \tilde{k}^{\sigma}\right] \\
& O_{2}^{\mu \nu \rho \sigma}(k)=\operatorname{Sym}\left[k^{4}\left(k^{\mu} k^{\rho}-\eta^{\mu \rho} k^{2}\right) \tilde{k}^{\nu} \tilde{k}^{\sigma}\right] \\
& O_{3}^{\mu \nu \rho \sigma}(k)=\tilde{k}^{2} k^{2}\left(k^{\mu} k^{\nu}-\eta^{\mu \nu} k^{2}\right)\left(k^{\rho} k^{\sigma}-\eta^{\rho \sigma} k^{2}\right) \\
& O_{4}^{\mu \nu \rho \sigma}(k)=\operatorname{Sym}\left[\tilde{k}^{2} k^{2}\left(k^{\mu} k^{\rho}-\eta^{\mu \rho} k^{2}\right)\left(k^{\nu} k^{\sigma}-\eta^{\nu \sigma} k^{2}\right)\right]
\end{aligned}
$$

where 'Sym' indicates normalized symmetrization in the index pairs $\mu \nu$ and $\rho \sigma$, and in $\mu \nu \leftrightarrow$ $\rho \sigma$.

\section{References}

[1] J. L. Hewett, F. J. Petriello, and T. G. Rizzo, Signals for non-commutative interactions at linear colliders, Phys. Rev. D64 (2001) 075012, hep-ph/0010354.

[2] I. Hinchliffe, N. Kersting, and Y. L. Ma, Review of the phenomenology of noncommutative geometry, Int. J. Mod. Phys. A19 (2004) 179-204, hep-ph/0205040.

[3] B. Jurco, L. Möller, S. Schraml, P. Schupp, and J. Wess, Construction of non-Abelian gauge theories on noncommutative spaces, Eur. Phys. J. C21 (2001) 383-388, hep-th/0104153.

[4] X. Calmet, B. Jurco, P. Schupp, J. Wess, and M. Wohlgenannt, The standard model on non-commutative space-time, Eur. Phys. J. C23 (2002) 363-376, hep-ph/0111115. 
[5] B. Melic, K. Passek-Kumericki, J. Trampetic, P. Schupp, and M. Wohlgenannt, The standard model on non-commutative space-time: Electroweak currents and Higgs sector, Eur. Phys. J. C42 (2005) 483-497, hep-ph/0502249.

[6] B. Melic, K. Passek-Kumericki, J. Trampetic, P. Schupp, and M. Wohlgenannt, The standard model on non-commutative space-time: Strong interactions included, Eur. Phys. J. C42 (2005) 499-504, hep-ph/0503064.

[7] T. Ohl and J. Reuter, Testing the noncommutative standard model at a future photon collider, Phys. Rev. D70 (2004) 076007, hep-ph/0406098.

[8] A. Alboteanu, T. Ohl, and R. Rückl, Probing the noncommutative standard model at hadron colliders, Phys. Rev. D74 (2006) 096004, hep-ph/0608155.

[9] A. Alboteanu, T. Ohl, and R. Rückl, The Noncommutative Standard Model at $O\left(\theta^{2}\right)$, Phys. Rev. D76 (2007) 105018, arXiv:0707.3595.

[10] L. Randall and R. Sundrum, A large mass hierarchy from a small extra dimension, Phys. Rev. Lett. 83 (1999) 3370-3373, hep-ph/9905221.

[11] N. Reshetikhin, Multiparameter quantum groups and twisted quasitriangular Hopf algebras, Lett. Math. Phys. 20 (1990) 331-335.

[12] C. Jambor and A. Sykora, Realization of algebras with the help of *-products, hep-th/0405268.

[13] P. Schupp and S. Solodukhin, Exact Black Hole Solutions in Noncommutative Gravity, arXiv:0906.2724

[14] T. Ohl and A. Schenkel, Cosmological and Black Hole Spacetimes in Twisted Noncommutative Gravity, JHEP 10 (2009) 052, arXiv:0906.2730.

[15] P. Aschieri and L. Castellani, Noncommutative Gravity Solutions, J. Geom. Phys. 60, 375 (2010), arXiv:0906.2774.

[16] P. Aschieri et. al., A gravity theory on noncommutative spaces, Class. Quant. Grav. 22 (2005) 3511-3532, hep-th/0504183.

[17] P. Aschieri, M. Dimitrijevic, F. Meyer, and J. Wess, Noncommutative geometry and gravity, Class. Quant. Grav. 23 (2006) 1883-1912, hep-th/0510059.

[18] T. Asakawa and S. Kobayashi, Noncommutative Solitons of Gravity, Class. Quant. Grav. 27, 105014 (2010), arXiv:0911.2136.

[19] A. Stern, Emergent Abelian Gauge Fields from Noncommutative Gravity, arXiv:0912.3021.

[20] M. R. Douglas and C. M. Hull, D-branes and the noncommutative torus, JHEP 02 (1998) 008, hep-th/9711165.

[21] V. Schomerus, D-branes and deformation quantization, JHEP 06 (1999) 030, hep-th/9903205. 
[22] N. Seiberg and E. Witten, String theory and noncommutative geometry, JHEP 09 (1999) 032, hep-th/9908142.

[23] M. Gunaydin, L. J. Romans, and N. P. Warner, Gauged N=8 Supergravity in Five-Dimensions, Phys. Lett. B154 (1985) 268.

[24] M. Gunaydin, L. J. Romans, and N. P. Warner, Compact and Noncompact Gauged Supergravity Theories in Five-Dimensions, Nucl. Phys. B272 (1986) 598.

[25] L. J. Romans, Gauged N=4 supergravities in five-dimensions and their magnetovac backgrounds, Nucl. Phys. B267 (1986) 433.

[26] H. Lu, C. N. Pope, and T. A. Tran, Five-dimensional $N=4$, SU(2) $x$ U(1) gauged supergravity from type IIB, Phys. Lett. B475 (2000) 261-268, hep-th/9909203.

[27] O. Aharony, J. Gomis, and T. Mehen, On theories with light-like noncommutativity, JHEP 09 (2000) 023, hep-th/0006236.

[28] L. Alvarez-Gaume, F. Meyer, and M. A. Vazquez-Mozo, Comments on noncommutative gravity, Nucl. Phys. B753 (2006) 92, hep-th/0605113.

[29] E. E. Boos, I. P. Volobuev, Y. A. Kubyshin, and M. N. Smolyakov, Effective Lagrangians of the Randall-Sundrum model, Theor. Math. Phys. 131 (2002) 629-640.

[30] E. E. Boos, Y. A. Kubyshin, M. N. Smolyakov, and I. P. Volobuev, Effective Lagrangians for physical degrees of freedom in the Randall-Sundrum model, Class. Quant. Grav. 19 (2002) 4591-4606, hep-th/0202009].

[31] H. Davoudiasl, J. L. Hewett, and T. G. Rizzo, Experimental probes of localized gravity: On and off the wall, Phys. Rev. D63 (2001) 075004, hep-ph/0006041.

[32] V. A. Kostelecky and M. Mewes, Signals for Lorentz violation in electrodynamics, Phys. Rev. D66 (2002) 056005, hep-ph/0205211].

[33] V. A. Kostelecky and N. Russell, Data Tables for Lorentz and CPT Violation, arXiv:0801.0287 